\title{
Encuesta de caracterización para el cuidado de una persona con enfermedad crónica'
}

\author{
Lucy Barrera Ortiz² \\ Elizabeth Vargas Rosero 3 \\ Paola Andrea Cendales 4
}

doi:10.11144/Javeriana.IE17-1.eccp

Cómo citar: Barrera Ortiz L, Vargas Rosero E, Cendales PA. Encuesta de caracterización para el cuidado de una persona con enfermedad crónica. Investig Enferm. Imagen Desarr. 2015;17(1):27-43. http://dx.doi.org/10.11144/Javeriana.IE17-1.eccp

1. Articulo original de investigación. Financiación: Programa para la Disminución de la Carga de la EC no Transmisible en Colombia. Código del programa en Colciencias: 501753730890. Recibido: 17 de julio de 2014. Aceptado: 1 de septiembre de 2014.

2. Enfermera. Especialista en Enfermería Pediátrica. Magíster en Enfermería. Profesora titular pensionada, Universidad Nacional de Colombia. Correo electrónico: 1barrerao@unal. edu.co

3. Enfermera. Magíster en Enfermería. Profesora asistente, Facultad de Enfermería, Universidad Nacional de Colombia. Correo electrónico: evargasr@unal.edu.co

4. Estudiante auxiliar del Proyecto Disminución de la Carga de la ECNT en Colombia. Correo electrónico: pacendalesr@unal.edu.co 


\section{Resumen}

Objetivo: Desarrollar la encuesta de caracterización para el cuidado de una persona con enfermedad crónica, a fin de utilizarla en el contexto colombiano. Método: Estudio metodológico que, a partir de la revisión de una propuesta de instrumento de caracterización anterior, y como parte del programa para la disminución de la carga de la enfermedad crónica en Colombia, determinó la información básica requerida para valorar a una persona con enfermedad crónica. Se desarrolló en tres fases: 1) revisión de la literatura relacionada con la provisión de cuidado a personas con enfermedad crónica; 2) definición de la encuesta de caracterización para el cuidado de una persona con enfermedad crónica, que se denominó GCPC-UN-P, y 3) validez aparente, que consistió en una prueba de campo con 1850 personas con enfermedad crónica, y una validez con expertos. Resultados: Se obtuvo la encuesta de caracterización GCPC-UN-P de 22 items que indagan acerca de tres dimensiones: las condiciones del paciente y su perfil sociodemográfico, la percepción de carga y apoyo, y los medios de información y comunicación. A la validez aparente la totalidad de los ítems son evaluados como comprensibles, claros, coherentes, suficientes, relevantes y esenciales. Conclusiones: La encuesta GCPC-UN-P cuenta con las variables necesarias para la caracterización de una persona con enfermedad crónica en el contexto colombiano.

Palabras clave: enfermedad crónica; encuesta socioeconómica; costo de enfermedad

\section{Characterization Survey to Define the Care of a Person with Chronic Disease}

\section{Abstract}

Objective: To develop the characterization survey to define the care of a person with chronic disease, in order to use it in the Colombian context. Method: Methodological study that determined the basic information required to assess a person with chronic disease after reviewing a previous proposal of a characterization tool and as part of the program for reducing the burden of chronic disease in Colombia. It was developed in three phases:1) Review of the literature related to the provision of care for people with chronic disease 2) definition of the characterization survey for the care of a person with chronic disease, which was named GCPC-UN-P, and 3) Apparent validity, which consisted of a field trial with 1850 people with chronic disease, and validity with experts. Results: It was obtained a characterization survey GCPC-UN-P of 22 items that ask about three dimensions: patient conditions and socio-demographic profile, perceived burden and support, and communication media. Regarding the apparent validity all items are evaluated as understandable, clear, coherent, adequate, relevant and essential. Conclusions: GCPC-UN-P has the necessary survey for the characterization of a person with chronic illness in the Colombian context variables.

Keywords:chronic disease; socioeconomic survey; disease cost 


\section{Inquérito sobre caracterização para o cuidado de uma pessoa com doença crônica}

\section{Resumo}

Objetivo: Desenvolver o inquérito de caracterização para o cuidado de uma pessoa com doença crônica, a fim de utilizá-la no contexto colombiano. Método: Estudo metodológico que, a partir da revisão de proposta de instrumento de caracterização anterior, e como parte do programa para diminuição da carga da doença crónica na Colômbia, determinou a informação básica requerida para valorar uma pessoa com doença crónica. Desenvolveu-se em três fases: 1) revisão da literatura relacionada com a provisão de cuidado a pessoas com doença crónica; 2) definição do inquérito de caracterização para o cuidado de uma pessoa com doença crónica, nomeada GCPCUN-P, e 3) validez aparente, que consistiu em um teste de campo com 1850 pessoas com doença crónica, e uma validez com peritos. Resultados: Obteve-se o inquérito de caracterização GCPC-UN-P de 22 itens que investigava acerca de três dimensões: as condições do paciente e seu perfil sociodemográfico, a percepção de carga e apoio, e os meios de informação e comunicação. À validez aparente a totalidade dos itens são avaliados como compreensiveis, claros, coerentes, suficientes, relevantes e essenciais. Conclusões: O inquérito GCPC-UN-P conta com as variáveis necessárias para a caracterização de uma pessoa com doença crónica no contexto colombiano.

Palavras chave: doença crónica; inquérito socioeconómico; custo de doença 


\section{Introducción}

La enfermedad crónica (EC) se está incrementando de manera acelerada y está ocasionando en la actualidad el $63 \%$ de las muertes en el mundo. Tal tendencia es especialmente marcada en los paises en desarrollo y resulta preocupante para América Latina, donde se espera el mayor impacto por esta situación. Colombia no es una excepción: la probabilidad de morir por una EC de los 30 a los 70 años de edad es cercana al $20 \%$, y como en los demás países de la región su tendencia va en ascenso (1).

La atención a las personas que viven la experiencia de cuidado en situaciones de EC, incluidos los pacientes, los familiares y los profesionales es demandante y requiere apoyo por parte de los administradores de las instituciones de salud para garantizar acceso, seguridad, continuidad, fortalecimiento de la habilidad de cuidar y apoyo social con uso de las tecnologías de información y comunicación (TIC) (2).

Algunas propuestas regionales de cuidado de estas personas han demostrado ser exitosas (3-5); sin embargo, se requiere tener unos mínimos en la valoración de la persona enferma, de manera que pueda responderse a sus necesidades de cuidado. Este trabajo presenta una propuesta de encuesta de caracterización para el cuidado de la persona con EC, desarrollada para el contexto colombiano.

\section{Método}

Estudio metodológico que, a partir de la revisión de una propuesta de caracterización del paciente con EC publicada en 2009 (6), y como parte del Programa para Disminución de la Carga de la EC en Colombia, buscó determinar las necesidades de información básicas para valorar a una persona que vive una condición de EC. Se desarrolló en tres fases:

1. Revisión de la literatura relacionada con la provisión de cuidado a personas con enfermedad crónica y necesidades de información requerida.

2. Definición de la encuesta de caracterización para el cuidado de una persona con enfermedad crónica, que se denominó GCPC-UN-P, por las siglas del Grupo de Cuidado al Paciente Crónico de la Universidad Nacional de Colombia-encuesta dirigida al paciente, versión 2014.

3. Validez aparente. Con la cual se determinó el grado en el cual los ítems parecen medir lo que se proponen (7). Para ello se realizó:

- Prueba de campo: por medio de la técnica de sondeo, de acuerdo con lo descrito por Arribas (8), para evaluar la validez de forma, equivalencia conceptual y aceptabilidad de la encuesta se administró en físico o virtualmente, con y sin apoyo de un entrevistador a personas con EC que voluntariamente aceptaron el diligenciamiento de esta.

- Validez con expertos: se conformó un grupo de seis expertos en cuidado al paciente crónico, con más de cinco años de experiencia en el área, y título de posgrado, con el fin de determinar si el instrumento realmente medía lo que se proponía medir. Estos expertos evaluaron los items en su claridad, coherencia, 
suficiencia, relevancia y esencia, según lo propuesto por Lawshe y descritos por Tristan (9). Adicionalmente, se contó con la asesoría de dos estadísticos y una psicóloga experta en psicometría.

\section{Resultados}

\section{Fase 1. Revisión de la literatura}

Se identificaron 38 referencias bibliográficas que se relacionan con la provisión de cuidado a personas con EC y necesidades de información requerida. Estas se organizaron en tres dimensiones que se reflejan en la encuesta. A continuación se describen los hallazgos de esta revisión.

\section{Dimensión 1. Identificación de condiciones del paciente y su perfil sociodemográfico}

Funcionalidad y estado cognitivo. Leung y colaboradores (10) reportaron que las personas enfermas tienen mayor riesgo de padecer sintomas psicológicos. Mediante un análisis multivariado señalaron que la función cognitiva, entre otras características, se asocia con una mayor proporción de síntomas depresivos y de ansiedad en quienes tienen EC.

Barrera y colaboradores (6) trataron de medir el grado de funcionalidad en pacientes y señalaron que la escala de valoración funcional PULSES (P: estabilidad de la patología; U: utilización de miembros superiores; L: locomoción; S: función sensorial; E: esfínteres, y S: capacidad de socializar) ha demostrado ser aplicable en diferentes edades. Las autoras reportaron que por ser esta una escala corta, sencilla y que puede ser administrada por el cuidador o por el profesional de salud, tiene amplia utilidad en la determinación de la dependencia de las personas con EC.

Rodríguez (11) señaló que en la prevención y el tratamiento de los adultos con EC se presenta falta de desarrollo de intervenciones psicoterapéuticas que pueden generar buenos resultados en el trabajo con los cuidadores. Diversos autores reportan que la funcionalidad reflejada en las actividades sociales, el sueño, el bienestar psicológico, la calma, los problemas físicos, entre otros, son ejemplos de aspectos que pueden afectar la calidad de vida del paciente, del cuidador y de la familia en general. Por ello, es importante conocer el grado de funcionalidad, para cuidar al paciente y, además, para prevenir el cansancio en el rol del cuidador que se asocia con la dependencia severa (12).

Uno de los instrumentos que se utilizan para valorar la funcionalidad mental es el Short Portable Mental Status Questionnaire (SPMSQ), que se trata de un test estandarizado para la exploración de la memoria episódica que ayuda a detectar la existencia de deterioro cognitivo en personas mayores de 65 años de edad.

Diagnósticos médicos. Barrera y colaboradores (6) indican que conocer el diagnóstico médico permite documentar las situaciones de EC 
dentro de los sistemas de salud. Esta información es clave para organizar grupos de apoyo con problemáticas similares. Rincón y colaboradores (13) han documentado que la EC significa un estado funcional deteriorado y la necesidad de continuidad de la atención. La coexistencia de múltiples condiciones crónicas en una sola persona representa una sobrecarga y, así, un mayor contacto con el sistema sanitario. La noción de polipatología difiere de muchos indices de comorbilidad, ya que su definición tiene en cuenta no solo la sumatoria de EC, sino también su importancia, en términos de estatus funcional o necesidad de un control estricto, incluso en ausencia de deterioro.

En el 2013, la Organización Mundial de la Salud (14) señalaba que alrededor del $15 \%$ de la población sufría de discapacidad y que el aumento de las EC no transmisibles tiene un profundo efecto en el incremento de estas tendencias. Conocer el(los) diagnóstico(s) médico(s) en cada caso permite estructurar el perfil epidemiológico respectivo y medir la carga de la enfermedad bajo estándares previamente acordados.

Género. Leung y colaboradores (10) documentaron diferencias de género con respecto a los sintomas de ansiedad y similitudes en los de depresión de personas con EC. Las mujeres mayores reportan tener peor función cognitiva y más riesgo de depresión; pero entre los sexos se encontró el mismo grado de apoyo social y de funcionamiento familiar. Por otra parte, se ha documentado que los hombres parecen percibir su salud peor que las mujeres en la presencia de EC (15).

Barrera y colaboradores (6), por su parte, refieren que los hombres son más apoyados por sus esposas, que a la inversa. Es preciso recordar que la mujer tiende a casarse con una persona mayor que ella y tiene más expectativa de vida, lo que afecta en muchos casos el nivel de apoyo que genera y el que recibe la pareja según el género.

Edad. A pesar de que la presencia de EC tiene aspectos comunes en todas las edades, tal como lo documentan Pinto (16), Barrera (17) y Sánchez (18), los problemas de cuidado se diferencian según el grupo etáreo afectado.

Escolaridad. La educación ha sido asociada con la prevención de las EC, pues se señala una relación positiva entre el nivel educativo y la conducta preventiva $(19,20)$. El nivel de escolaridad ha mostrado una relación inversa con la prevalencia de obesidad, hipertensión y diabetes, lo que se traduce en un mayor promedio de factores de riesgo de EC en grupos educación básica y media incompleta (21). Más aún, hay reportes de mayor prevalencia de EC entre hombres y mujeres viejos cuando estos tienen menor nivel de escolaridad (15). Se ha demostrado también que los niveles básicos de educación proporcionan el fortalecimiento de relaciones personales, sociales e integración social. Por ejemplo, saber que una persona puede leer facilita el manejo de los medicamentos y de otros aspectos de cuidado que muchas veces son comunicados mediante material escrito (6).

Procedencia. Bien sea esta rural o urbana, incide de muchas formas en la presencia de EC. Diversos estudios asocian las diferentes regiones 
del país a la aparición o frecuencia de algunas EC. Por ejemplo, Bernal y colaboradores (22) encontraron que hubo una mayor incidencia de enfermedades no transmisibles en Colombia en el quinquenio 2004-2008, especialmente en áreas urbanas y centrales del país; mientras que en los departamentos selváticos y costeros se observó una preponderancia de patologías transmisibles.

Lugar de residencia. Al estudiar a personas mayores que viven en comunidades rurales, se encontró que tuvieron significativamente menos ansiedad, en comparación con las personas de edad avanzada que viven en una comunidad urbana (10). Los factores ambientales han sido determinantes en al menos la cuarta parte de la carga de la morbilidad en las personas en el mundo (23).

Estado civil. Contar con una pareja es un factor de ayuda para muchos cuidadores, cuando se deben tomar decisiones difíciles. Sin embargo, la solicitud demandante de un compañero dificulta el desempeño del rol. En la relación entre esposos, cuando uno de los miembros de la pareja sufre un deterioro de la salud y necesita ayuda para sus actividades de la vida diaria, el cuidador principal suele ser el de mejor salud, pero en el proceso puede terminar deteriorándose (6). Por otra parte, se ha reportado que la unión de pareja es una garantía para el hombre, pero no siempre lo es para la mujer (24); no obstante, es importante tener en cuenta que no hay documentación suficiente respecto a los cuidadores hombres en el mundo.

Ocupación. En el 2010 se estudió la proporción de personas con EC con síntomas físicos o discapacidad que tenían trabajo remunerado por al menos 12 horas a la semana y se encontró en el $48 \%$ de aquellos entre los 15 y los 39 años; en el 38\% entre los 40 y los 54 años, y en el 16\% entre los 55 y los 64 años. La mala salud se asoció con baja participación y la EC con la ausencia de trabajo remunerado (25). La discapacidad relacionada con la EC no transmisible como amputación, ceguera o parálisis pone significativas exigencias a los sistemas de salud y bienestar social, al disminuir la productividad y empobrecer a las familias (14). De hecho, las medidas de carga de la EC en el mundo se generan alrededor de los años de vida productiva perdidos (26-30).

Estrato socioeconómico. Aunque la EC se asocia con secuelas de violencia, pobreza y contaminación, esta no respeta condiciones sociales (6). Algunos estudios han demostrado que si bien hay una mayor tendencia a asociar algunos comportamientos que favorecen la aparición de la EC en estratos sociales bajos, estos no son exclusivos de estos grupos (31).

Tiempo que lleva con la EC. Como su nombre lo indica, la EC es prolongada en el tiempo y se caracteriza por tener diferentes etapas o fases (32). Las diferencias de percepciones cambian en los diferentes periodos; sin embargo, las variaciones más importantes de la experiencia se presentan en los primeros seis meses (6).

Religión. En el transcurso de la EC, las personas cambian la relación con ellas mismas, se comunican con Dios o un ser o fuerza superior y tienen una relación diferente con otras personas y con el medio. La experiencia encierra múltiples significados e invita a cuestionar o a ratificar los 
propios valores. La espiritualidad -en particular el componente religioso- puede influir en el afrontamiento general a la EC, en el dar o recibir cuidado, en la disminución del sentimiento de carga, en incrementar la habilidad y en acentuar los beneficios de la experiencia $(33,34)$.

\section{Dimensión 2. Percepción de carga y de apoyo}

Cuidador único. Tener un cuidador modifica la experiencia de vivir con una EC y, en algunos casos, permite generar un vínculo especial e insoluble que transforma el sentido de la vida (35). Aunque la relación entre la edad avanzada y la enfermedad hace que en la mayoría de casos los cuidadores sean menores que la persona cuidada, esto no siempre es así. Tener un cuidador es un factor que afecta la experiencia de vivir en condición de EC. Cuando el cuidador es muy joven, las actividades pueden ser muy bien desarrolladas por su habilidad física, pero el cuidado se ofrece a instancias del desarrollo de la personalidad. Los menores suelen tener mayor valor para arriesgarse y menor constancia que los de más edad. Entre los 18 y los 35 años, los cuidadores tienden a ver frustradas las metas proyectadas para sus vidas. Muchos de ellos están iniciando la crianza de los hijos, lo que ocupa la mayor parte de sus energías y el cuidado del enfermo compite con su responsabilidad principal o cuando se cuida al hijo agobia de manera especial. Entre los 36 y los 59 años, el grupo de cuidadores conforma lo que se ha denominado generación sándwich. La tarea de cuidador se da de forma simultánea con el rol de trabajador(a) y, a veces, con el cuidado de los hijos. Es preciso recordar que en la cultura latina el joven permanece en su hogar hasta que se casa o se independiza, después de ser económicamente solvente. En el caso de los cuidadores mayores, se observa que, con el proceso de envejecimiento, empiezan a tener una salud más vulnerable que se puede ver afectada debido a la carga del cuidado (6).

Horas de cuidado requeridas. Las horas diarias que precisa una persona con EC reflejan su grado de dependencia y se asocian con la carga del cuidado. Estas han sido utilizadas para determinar la carga que genera la situación de EC (36). Se ha documentado que la soledad se asocia con la dependencia en las actividades de la vida diaria, lo que se expresa en el tiempo requerido para el cuidado (37).

Vínculo con la persona que lo cuida. En América Latina, la mayor parte de las personas con EC son atendidas por sus familiares (6). Las características de estos cuidadores se han asociado con la percepción del estado de salud (38). La participación familiar puede amortiguar los efectos de los sintomas psicológicos en personas con deterioro cognitivo y otras enfermedades. Es decir, la familia es una fuente importante de apoyo para estas personas y debe ser una de las variables de apoyo social en los estudios (10).

Por otra parte, la conducta del hombre cuidador tiende a ser más racional, y la de la mujer cuidadora, más afectiva. La cultura latina hace que el rol del cuidado lo asuman con mayor facilidad las mujeres, quienes buscan con mayor detalle proporcionar un mejor estilo y calidad de vida 
a la persona cuidada. Sin embargo, los hombres a cargo de una persona con EC suelen ser más escuchados en las situaciones difíciles del cuidado. Los hijos tienen una relación de cuidado más perdurable y la pareja o los familiares son más acogidos que los amigos, vecinos o instituciones. El cuidado de hijos a padres, en especial ancianos, representa un fuerte impacto emocional al asumir una inversión de roles (6).

Apoyos con los que cuenta y satisfacción con ellos. Los apoyos con que cuente la persona con EC determinan de manera contundente la forma en que vive la experiencia. Al respecto, Yeh (37) señala que la insatisfacción con el apoyo social, el tamaño de la red de apoyo y el estado funcional son predictores significativos de soledad en los adultos mayores con EC. Para muchos de ellos, el apoyo emocional es más importante que el apoyo instrumental. En ausencia de acontecimientos estresantes, una amplia red social puede promover la salud.

Por otro lado, en presencia de estrés significativo, el apoyo funcional puede ser importante para mejorar las tensiones. El apoyo instrumental y el emocional se encuentran alta y están inversamente relacionados con la depresión y los sintomas de ansiedad. Existe un efecto amortiguador moderado entre la participación familiar y los síntomas psicológicos que se asocian con la capacidad de los sujetos para afrontar el deterioro cognitivo y las EC (10).

Respecto al apoyo del cuidador familiar, la percepción de demasiada carga para el cuidador también repercute en cómo la persona cuidada se percibe a sí misma. Los cuidadores que tienen un elevado grado de satisfacción con el apoyo social, por parte de la familia, los amigos y los profesionales, bien sea emocional o instrumental, experimentan menos efectos negativos de las crisis. Por el contrario, la soledad y la falta de apoyo generan sufrimiento que, en definitiva, afecta a la persona con EC (6).

Es de anotar que tanto el paciente con EC como su cuidador que tienen un elevado grado de satisfacción con el apoyo social por parte de la familia, los amigos y los profesionales, bien sea emocional o instrumental, experimentan menos efectos negativos de las crisis. Ellos también sostienen que la soledad y la falta de apoyo les generan sufrimiento (6).

Ahora bien, la satisfacción con la vida se ha relacionado también con el sentido de control, la autoestima, el sentimiento de certeza sobre el futuro, la percepción de conexión, los recursos sociales disponibles, el no uso de drogas psicotrópicas y la ausencia de sentimientos negativos como frustración, depresión, carga o ambivalencia respecto a la situación.

Las personas cuidadoras y las receptores describen profunda pena, asociada a la discapacidad que otros no comprenden. Frente a ello los sistemas de apoyo social público y privado continúan distantes de las necesidades y son insuficientes para dar esperanza en la vida diaria o para proporcionar una tregua que evite el cansancio y la fatiga con la labor de dar y recibir cuidado (39).

Investigaciones que han medido las repercusiones de la enfermedad crónica en quienes la padecen han encontrado que el hecho de que estas personas perciban un bajo apoyo por parte de sus familiares o amigos incrementa sus cifras de morbilidad y mortalidad y generan aislamiento social (40). 
Finalmente, la familia se identifica como la principal fuente de apoyo en diferentes contextos. Se ha demostrado que la participación familiar amortigua algunos efectos de los sintomas del paciente, en especial los psicológicos, y que incide más en los comportamientos saludables que en el apoyo social general. Por ello una mejor comprensión del funcionamiento de la familia y de la salud puede ofrecer explicaciones más profundas de sus efectos en el cuidado de la salud (41).

Percepción de carga para la familia y de bienestar. La dedicación exclusiva a los cuidados por parte del cuidador implica una restricción de su vida social, y si a esto se suman situaciones de menor funcionalidad de la persona a quien se cuida, se experimenta mayor carga y sentimientos de depresión, lo cual termina repercutiendo en la persona con EC (6). Se ha encontrado que la percepción de carga de la persona con EC se asocia a su bienestar espiritual (42). También que el bienestar emocional predice el pronóstico a largo plazo de la enfermedad física. Varios estudios indican que el bienestar está asociado positivamente con una mejor salud (43).

\section{Dimensión 3. Medios de información y comunicación}

Autovaloración en el uso de TIC. Las restricciones que pueden llegar a tener las personas que viven con EC son importantes y requieren apoyo para garantizar acceso, seguridad, continuidad, fortalecimiento de la habilidad de cuidar y apoyo social con uso de las TIC. Un estudio nacional reciente documenta que, a pesar de las posibilidades que dan las TIC para apoyar a estas personas, el grado de conocimiento y de acceso continúan siendo medio y bajo entre ellas y es heterogéneo entre las diferentes regiones del país (44). Se complementa esto con hallazgos anteriores que reportan una buena aceptación de las TIC por parte los cuidadores y administradores de servicios de salud (45).

Disponibilidad de TIC para apoyar el cuidado. Valorar el apoyo que ofrecen o pueden ofrecer las TIC para el cuidado de las personas con EC da caminos para hacerlo operativo. Los mayores beneficios identificados incluyen la valoración positiva del apoyo social, el autorreconocimiento, el crecimiento personal, la sensación de tener un vínculo y la comunicación misma que genera utilidad y satisfacción con las respuestas (46). El apoyo social logrado a través del uso de TIC genera efectos positivos tanto para el cuidador como para el sujeto de cuidado, fortalece el vínculo afectivo y mejora la unidad familiar, pues brinda elementos que disminuyen el estrés, la depresión, la soledad y el aislamiento, que en la mayoría de los casos genera la EC (45).

Apoyo percibido con el uso de TIC. El cuidado con uso de TIC ha demostrado ser apreciado por las personas con EC en la atención de salud en el hogar, pues mejora la sensación de seguridad en su vida cotidiana y el funcionamiento de la vida diaria. Las TIC les permiten estar en mayor contacto con el medio que los rodea y facilitan la independencia en algunos 
aspectos de su vida cotidiana cuando tienen acceso y saben cómo hacer uso de ellas (47).

Utilidad de las TIC para recibir información del cuidado. La utilidad de las TIC es relativa a las necesidades y a las capacidades de la persona que las emplea. En tal sentido, se ha señalado la necesidad de continuar identificando y diseñando TIC acordes al contexto de los usuarios, de explorar más algunas herramientas como internet y de profundizar en las redes de apoyo social en línea para el cuidado de la salud de las personas con EC (48).

El apoyo telefónico, por ejemplo, ha demostrado ser útil frente al cuidado emocional y a la posibilidad de dar información con sensibilidad cultural, ya que permite mantener una conexión humana, la expresión de sentimientos y el apoyo en aspectos relacionados integralmente con la salud. En intervenciones por internet para ancianos y sus cuidadores, las redes sociales tienen ventajas como la disponibilidad las 24 horas del dia y el anonimato, que puede conducir a una comunicación más desinhibida y franca. Algunas barreras para proporcionar este tipo de intervenciones son el estigma, la falta de tiempo, el transporte cuando es necesario un encuentro presencial y la falta de conocimiento sobre el servicio (45).

En cuanto a los grupos de apoyo en línea, se han reportado limitaciones como no contar con un computador, módem o software de comunicación; la falta de acceso a una cuenta de internet, o tener dichos recursos en idioma diferente al propio (45). El uso de las TIC con video a través de una conexión de banda ancha es un apoyo que permite mejorar la calidad de vida de las personas mayores o enfermas que viven en su casa. Un programa de mensajería facilita la comunicación entre la enfermera y las personas con EC que tienen dificultades para hablar (47).

Por ello es fundamental atender la calidad de la información, lo que es coherente con la tendencia actual de certificar los portales en internet a través de la Organización Mundial de la Salud o sus asociados, por ejemplo, HONcode (http://www.hon.ch) y bases de datos cientificas en salud reconocidas.

\section{Fase 2. Definición de la encuesta}

Con base en la revisión de la literatura, se compararon cada uno de los items de la encuesta publicada en el 2009. Este proceso generó la inclusión de siete ítems, la redefinición de cinco y la organización de estos en tres dimensiones: 1) condiciones del paciente y su perfil sociodemográfico, 2) percepción de carga y apoyo y 3) medios de información y comunicación.

\section{Fase 3. Validez aparente}

Prueba de campo. Participaron 1850 personas con EC en las cinco regiones geográficas de Colombia: Amazonía, Andina, Caribe, Orinoquía y 
FCHA DE CARACTERZACION DE LA PERSOMA CON ENFERMEDAD CRONICA GCPC-UNPA Institución en la que se dligencia esta ficha: Nombre del Paciente: Teléfono: _Correo electrónico:

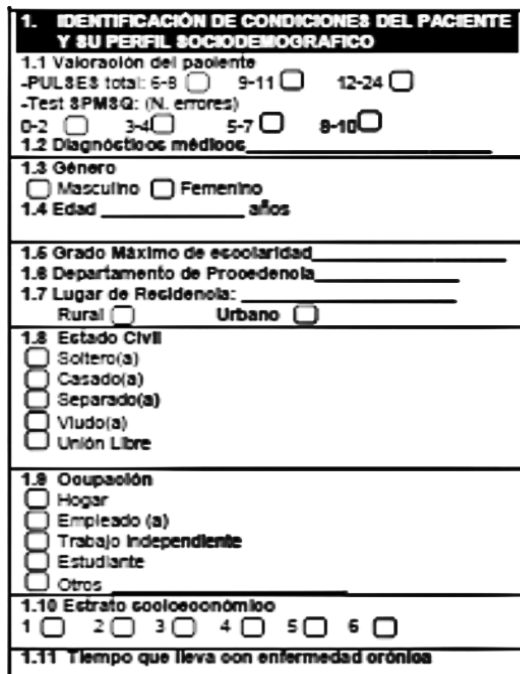

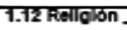

Nivel de Compromlso Religloso

$\square$ A:o $\square$ Medio $\square$ Bajo

\section{PERCEPCIOM DE CARGA Y DE APOYO \\ 21 Thene ur}

Si $\square$ No $\square$

Edad dol (loc) ouldador(oc):

22 Namero de horac de ayuda que requlere

dlariamento para cu culdado

23 La percona que lo culda ec:

Esposo (a)

Madre:padre

QHola)

Grueio(a)

Qumbola)

Nombre de la persona que diligencla el instrumentio correo electronico:
2.4 indlque oon una $x$ loc apoyoc con loc que ouenta y alifique eu nivel do catictacolon oon recpeoto a loc micmoc, clendo 1 el minimo y 4 ol maximo:

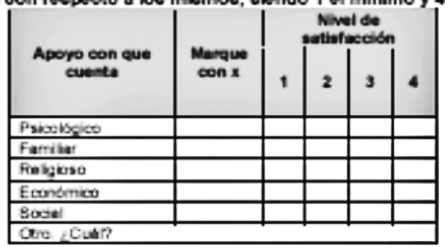

26 Porobe que para cu famlla, uctod oomo paciente ec una oaroa: Muy Als $\square$ Ats $\square$ Moderads $\square$ Bala $\square$

28 Al mirar cu oondiolion y colldad de vida aotusiloc, ocmo oalinoa loe 2. A mirar cu oondiolion y calldad do vida aotusioc, $00 \mathrm{mo}$ oalinoa blenectar pocible:

$$
\begin{array}{|l|l|l|l|l|l|}
\cline { 3 - 6 } & 0 & 1 & 2 & 3 & 4 \\
\hline
\end{array}
$$

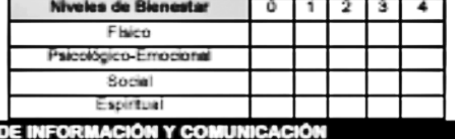
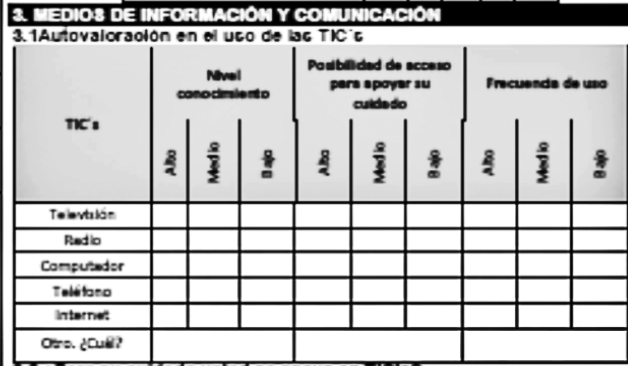

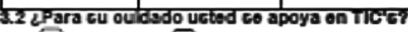

SI $\square$ No $\square$

3.3 Nivel de apoyo perolbido ocen el ueo do Ticte en el ouldado de eu enfermedad

Nito $\square$ Medo $\square$ Bajo $\square$

34 Ordene per pritorided lec TIC'c que lec podrian cer mac utlec para reolbir intormaolon para cu ouldado, clondo 1 ol menoc utall y 6 ol mate util:

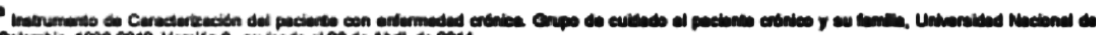

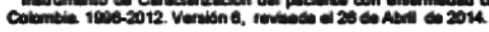

Figura 1. Encuesta GCPC-UN-P versión 2014

Fuente: elaboración propia
Telefono:

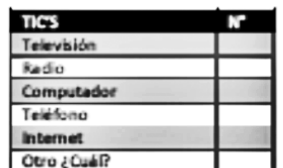


Pacífico, cuya población se caracteriza por aspectos comunes en lo referente a las costumbres socioculturales y familiares (49). Todos los items de la encuesta obtuvieron una calificación del 100\% de comprensibilidad y claridad, dada por los participantes. El tiempo para su diligenciamiento estuvo entre 10 y 20 minutos. Durante la aplicación no se requirieron nuevos ajustes. La información resultante de la caracterización es reportada en otro escrito. La versión final de la encuesta se presenta en la figura 1. Como recomendación se consideró necesario elaborar un instructivo para orientar a quienes la pretendan aplicar.

Validez de expertos. Los expertos valoraron el $100 \%$ de los items como claros, coherentes, suficientes, relevantes y esenciales para cada una de las dimensiones a las que pertenecían. Como recomendaciones se determinó la importancia de desarrollar un aplicativo virtual para la aplicación de la encuesta.

\section{Conclusiones}

La encuesta de caracterización GCPC-UN-P incluye 22 items organizados en tres grandes categorias: condiciones del paciente y su perfil sociodemográfico; percepción de carga y apoyo, y medios de información y comunicación. En conjunto, esta encuesta cuenta con las variables necesarias para conocer aspectos relacionados con un cuidado de calidad para quienes viven situaciones de EC (2). Por último, demostró ser aplicable en el contexto colombiano.

\section{Limitaciones}

Se consideran limitaciones del estudio la escasa literatura sobre metodologías descritas formalmente para realizar cada uno de los procesos de construcción y validación de una encuesta de caracterización, por lo que se restringe la realización de pruebas psicométricas como validez de constructo, entre otras.

\section{Referencias}

1. Organización Mundial de la Salud. Estadísticas sanitarias mundiales 2012. Ginebra: OMS; 2012.

2. Arias M, Sánchez B. Las cinco características que una institución requiere para dar cuidado de calidad a personas con enfermedad crónica. Documento procedente del I Congreso Virtual-IX Reunión Internacional de Enfermería Basada en la Evidencia. Cuidados y Tecnología: Una Relación Necesaria. Granada, España; 21-22 noviembre 2013.

3. Barrera L, Pinto N, Sánchez B. Evaluación de un programa para fortalecer a los cuidadores familiares de enfermos crónicos. Rev Salud Pública. 2006;8(1):141-52.

4. Barrera L, Pinto N, Sánchez B, Galvis C, Moreno M, Pinzón M, Romero E. La habilidad de cuidado de los cuidadores familiares de personas con enfermedad crónica: un estudio comparativo en 
tres unidades académicas de enfermería. Invest Educ Enferm. 2006;24(1):36-46.

5. Pinto N, Barrera L, Sánchez B, Figueroa P, Camargo L. Habilidad de cuidado de los cuidadores familiares de personas con enfermedad crónica: mirada internacional. Aquichán. 2006;6(1):22-33.

6. Barrera L, Pinto N, Carrillo G, Chaparro L, Sánchez B. Cuidado a los cuidadores familiares de personas con enfermedad crónica. Bogotá: Universidad Nacional de Colombia; 2010.

7. Carvajal A, Centeno C, Watson R, Martínez M, Sanz Á. ¿Cómo validar un instrumento de medida de la salud? Anales Sis San Navarra [internet]. 2011;34(1):63-72. Disponible en: http://scielo.isciii.es/scielo. php?script=sci_arttext\&pid=S1137-66272011000100007\&lng=es.

8. Arribas A. Adaptación transcultural de instrumentos: guía para el proceso de validación de instrumentos tipo encuestas. Revista Científica de la AMBB. 2006;16(3):74-82.

9. Tristán A. Modificación de los modelos de Lawshe para el dictamen cuantitativo de la validez de contenido de un instrumento objetivo. Avances en Medición. 2008;6:37-48.

10. Leung K, Chen C, Lue B, Bee-Horng L, Shih T. Social support and family functioning on psychological symptoms in elderly Chinese. Arch Gerontol Geriatr. 2007;44(2):203-13.

11. Rodríguez E. Effectiveness of an intervention in groups of family caregivers of dependent patients for their application in primary health centers. Study protocol. BMC Public Health. 2010;10:559.

12. Flores E, Rivas E, Seguel F. Nivel de sobrecarga en el desempeño del rol del cuidador familiar de adulto mayor con dependencia severa. Cienc Enferm. 2012;18(1):29-41.

13. Rincón M, Bernabeu M, Bohórquez P, Moreno L, Cassani M, Ortiz M, Ollero M. Perceived quality of healthcare in a multicenter, community-based population of polypathological patients. Arch Gerontol Geriatr. 2011;52:142-6.

14. World Health Organization. Global action plan for the prevention and control of noncommunicable diseases 2013-2020. Washington; 2013.

15. Barreto S, Figueiredo R. Doença crônica, auto-avaliação de saúde e comportamento de risco: diferença de gênero. Rev Saúde Pública. 2009;43(Suppl 2):38-47.

16. Pinto N. La cronicidad y el cuidado familiar, un problema de todas las edades: los cuidadores de adultos. Av Enferm. 2004;12(1):56-60.

17. Barrera L. La cronicidad y el cuidado familiar, un problema de todas las edades: los cuidadores de niños con enfermedad crónica. Av Enferm. 2004;12(1):49-55.

18. Sánchez B. La cronicidad y el cuidado familiar, un problema de todas las edades: los cuidadores de ancianos. Av Enferm. 2004;12(1):61-7.

19. Lucumí D, Gómez L. Accesibilidad a los servicios de salud en la práctica de citología reciente de cuello uterino en una zona urbana de Colombia. Rev Esp Salud Pública. 2004;78(3):367-77. 
20. Lewis MJ. Análisis de la situación del cáncer cervicouterino en América Latina y el Caribe. Washington, D.C.: OPS; 2004 [citado 2012 jun 29]. Disponible en: http://www.paho.org/.

21. Fagalde M, Solar J, Guerrero B, Atalah E. Factores de riesgo de enfermedades crónicas no transmisibles en funcionarios de una empresa de servicios financieros de la Región Metropolitana. Rev Méd Chile. 2005;133(8):919-28.

22. Bernal O, Forero JC, Villamil M, Pino R. Disponibilidad de datos y perfil de morbilidad en Colombia. Rev Panam Salud Publica. 2012;31(3):181-7.

23. Prüss-Üstün A, Ambientes saludables y prevención de enfermedades: hacia una estimación de la carga de morbilidad atribuible al medio ambiente [internet]. Ginebra: Organización Mundial de la Salud; 2006. Disponible en: http://www.who.int/quantifying_ehimpacts/publications/prevdisexecsumsp.pdf.

24. Verschuren J, Enzlin P, Dijkstra P, Geertzen J, Dekker R. Chronic disease and sexuality: a generic conceptual framework. J Sex Res. 2010;47(2):153-70.

25. Rijken M, Spreeuwenberg P, Schippers J, Groenewegen P. The importance of illness duration, age at diagnosis and the year of diagnosis for labour participation chances of people with chronic illness: results of a nationwide panel-study in the Netherlands labour market. BMC Public Health. 2013;13:803.

26. Ministerio de Salud de Chile, Pontificia Universidad Católica de Chile. Informe final estudio de carga de enfermedad y carga atribuible, Chile 2007. Santiago de Chile; 2007.

27. Rodríguez J, Acosta N, Peñaloza R. Carga de enfermedad Colombia 2005: resultados alcanzados [documento técnico]. Bogotá: CENDEX; 2008.

28. Lozano R, Franco MF, Solís P. El peso de la enfermedad crónica de México. Salud Pública Méx. 2008;55(3):301-9.

29. Borruel MA, Mas IP, Borruel GD. Estudio de carga de enfermedad: Argentina. Buenos Aires: Ministerio de Salud de la Nación; 2010.

30. Lozada P, Aguinaga L, Páez R, Olmedo C, Pozo A. El peso de la enfermedad en Ecuador [internet]. 1995. Disponible en: http://www. opecu.org/bevestre/revistas/CEPAR/pesoenf.pdf.

31. Secretaría de Salud de Santander, Observatorio de Salud Pública. Factores de riesgo para enfermedades crónicas en Santander [internet]; 2011. Disponible en: http://www.who.int/chp/steps/2010_ STEPS_Survey_Colombia.pdf.

32. Corbin J, Strauss A. A nursing model for chronic illness management based upon the trajectory framework. Sch Ing Nurs Prac.1991;5(3):155-74.

33. Peterman A, Fitchett G, Brady M, Hernández L, Cella D. Measuring spiritual well-being in people with cancer: the functional assessment of chronic illness therapy--Spiritual Well-being Scale (FACIT-Sp). Ann Behav Med. 2002;24(1):49-58. 
34. Riley B, Perna R, Tate DG, Forchheimer M, Anderson C, Luera G. Types of spiritual well-being among persons with chronic illness: their relation to various forms of quality of life. Arch Phys Med Rehabil. 1998;79(3):258-64.

35. Chaparro L. Cómo se constituye el "vínculo especial" de cuidado entre la persona con enfermedad crónica y el cuidador familiar. Aquichán. 2011;11(1):17-22.

36. Moya P, Escribano F, Pardo I, Notario B, Alfaro C, Martínez V. Costes asociados a las horas de cuidado informal de los mayores dependientes en un ámbito rural. Gac Sanit [internet]. 2009;23(2):109-14. Disponible en: http://scielo.isciii.es/scielo. php?script=sci_arttext\&pid=S0213-91112009000200006\&lng=es.

37. Yeh S. Living alone, social support, and feeling lonely among the elderly. Social Behavior and Personality. 2004;32(2):129-38.

38. Moreno ML, Nader A, López de Mesa C. Relación entre las características de los cuidadores familiares de pacientes con discapacidad y la percepción de su estado de salud. Av Enferm. 2004;22(1):27-38.

39. Sánchez B. Dimensión espiritual del cuidado de enfermería en situaciones de cronicidad y muerte. Bogotá: Editorial Universidad Nacional de Colombia; 2004.

40. Arechabala M, Catoni M, Palma E, Barrios S. Depresión y autopercepción de la carga del cuidado en pacientes en hemodiálisis y sus cuidadores. Rev Panam Salud Pública. 2011;30(1):74-9.

41. Leung K, Chen C, Lue B, Bee-Horng L, Shih T. Social support and family functioning on psychological symptoms in elderly Chinese. Arch Gerontol Geriatr. 2007;44(2):203-13.

42. Ballew S, Hannum S, Gaines J, Marx K, Parrish J. The role of spiritual experiences and activities in the relationship between chronic illness and psychological well-being. J Relig Health. 2012;51:1386-96-

43. Lamers S, Bolier L, Westerhof G, Smit F, Bohlmeijer E. The impact of emotional well-being on long-term recovery and survival in physical illness: a meta-analysis. J Behav Med. 2012;35:538-47.

44. Carrillo G, Chaparro L, Sánchez B. Conocimiento y acceso a las TIC en personas con enfermedad crónica y cuidadores familiares en Colombia. Enfermería Global. 2014;13(2):123-34.

45. Cárdenas D, Melenge B, Pinilla J, Carrillo G, Chaparro L. Soporte social con el uso de las TIC para cuidadores de personas con enfermedad crónica: un estado del arte. Aquichán. 2010;10(3):204-13.

46. Carrillo G, Chaparro L, Barrera L, Pinto N, Sánchez B. El blog como herramienta de soporte social para personas con enfermedad crónica. Cienc Enferm. 2011;17(3):137-49.

47. Nilsson C, Öhman M, Söderberg S. Information and communication technology in supporting people with serious chronic illness living at home: an intervention study. J Telemed Telecare. 2006;12(4):198-202.

48. Barrera L, Carrillo G, Chaparro L, Pinto, Sánchez B. Soporte social con el uso de las TIC's para cuidadores familiares de personas con enfermedad crónica. Rev Salud Pública. 2011;13(3):446-57. 
49. Gutiérrez de Pineda V. Familia y cultura en Colombia: tipologías, funciones y dinámica de la familia. Manifestaciones múltiples a través del mosaico cultural y sus estructuras sociales. Vol. 3. Bogotá: Instituto Colombiano de Cultura; 1975. 